\title{
Spectral calibration of the MSFC Solar Ultraviolet Magnetograph
}

\author{
Edward West ${ }^{1}$, Ken Kobayashi ${ }^{2}$, Jonathan Cirtain ${ }^{1}$, Allen Gary ${ }^{2}$, \\ John Davis ${ }^{1}$ and Joseph Reader ${ }^{3}$ \\ ${ }^{1}$ Space Science Office, VP62, NASA Marshall Space Flight Center, Huntsville, AL 35812 \\ ${ }^{2}$ The University of Alabama, Huntsville, CSPAR, Huntsville, AL 35899 \\ ${ }^{3}$ National Institute of Standards and Technology, Gaithersburg, MD 20899
}

\begin{abstract}
This paper describes the scientific goals of a sounding rocket program called the Solar Ultraviolet Magnetograph Investigation (SUMI), presents a brief description of the optics that were developed to meet those goals and discusses the spectral, spatial and polarization characteristics of SUMI's Toroidal Variable-Line-Space (TVLS) gratings, which are critical to SUMI's measurements of the magnetic field in the Sun's transition region.
\end{abstract}

Keywords: UV polarization, Solar Magnetograph, Sun, Sounding Rocket, Toroidal gratings, TVLS gratings

\section{INTRODUCTION}

The Solar Ultraviolet Magnetograph Investigation, SUMI, has been reported in several papers since this program began in $2000^{8,9,10}$. The emphasis in this paper will be to reference and summarize the test results presented in those papers while documenting the spectral alignment of SUMI's two toroidal variable-line-space (TVLS) gratings.

Section 2 reviews the scientific goals of SUMI and why this mission is important in understanding the 3D structure of the magnetic field on the Sun. The flight hardware that makes up the SUMI payload is described in Section 3 with emphasis on those components that affect the alignment of the TVLS gratings. Section 4 emphasizes the testing of our sounding rocket that is attached to a custom vacuum ultraviolet (VUV) collimator which provides the vacuum interface and wavelength calibration. With this VUV collimator we are able to verify that SUMI can achieve its spatial, wavelength and polarization resolution and meet its scientific goals.

\section{SCIENCE GOALS}

This section describes the importance of direct magnetic field measurements at various heights in the solar atmosphere and how the SUMI sounding rocket program plans to verify that VUV transition region polarization measurements are possible. While the SUMI sounding rocket program is a proof-of-concept mission, the goal of this program is to obtain high resolution CIV spectro-polarimetry data of a large unipolar sunspot or simple bipolar active region so that this data can be compared to the theoretical models and NLTE radiative codes that are being developed for this important transition region line $e^{1,2}$. While a large simple sunspot may not produce the polarization maps required to understand complicated structures in the transition region, SUMI's first flight is an essential step in developing the analysis tools needed to connect the photospheric and low chromospheric magnetic fields to those observed in the upper transition region.

\subsection{Magnetic field measurements in the transition region}

The problem of how energy, stored in the solar magnetic field, is released to heat the corona and drive the dynamic phenomena of the outer atmosphere, flares and coronal mass ejections, remains unsolved. Neither theoretical or numerical models nor current observations are able to provide a conclusive explanation. We have learned that a substantial fraction of this energy propagates outward into the interplanetary medium in the form of EM radiation, energetic particles, and the solar wind that create, control and modify the structure of the interplanetary medium. These 
products constitute space weather and have been shown to affect the immediate geo-space environment and create hazards for terrestrial and space assets. In the future, these will be a major concern for astronauts working on the lunar surface and traveling through interplanetary space. The solar input is the starting point for any model that describes and predicts space weather. Therefore understanding the processes that contribute to both the steady and transient release of magnetic energy into the solar atmosphere is a crucial element of these studies.

To understand the fundamental processes that lead to the explosive release of magnetic energy in solar flares and coronal mass ejections (CMEs), it is necessary to observe and infer the topology of the magnetic field and how it evolves prior to and during the energy release process. Success has been achieved in the characterization of the full vector field in the photosphere, where $\beta$, the ratio of the gas pressure to the magnetic pressure, is $>1$. At higher levels in the atmosphere (Figure 1$)^{3}$ where $\beta<1$, the magnetic field, through the Lorentz force, controls the structure and dynamics of the solar atmosphere, and rapid changes in its structure can produce energetic events. However, observations of the magnetic field at these higher levels have proven to be difficult, placing a serious limitation on our understanding of the physical processes occurring there.

The missing element is the ability to understand and describe quantitatively the transition layer where the field is no longer pressure-dominated as in the photosphere. The need to understand the energy release processes was identified two decades ago ${ }^{4}$, and was reaffirmed as the major conclusion of a workshop on the definition of the scientific goals for "Beyond Solar-B" (Moore, Davis, and Hathaway, 2001) $)^{5}$. To understand this process, full vector magnetic field measurements at several different heights in the solar atmosphere, from the photosphere to the upper transition region where the field becomes nearly force free, will be required. The vector field in the photosphere and low chromosphere has been measured with ground- based instruments. Space-based instruments such as the Michelson Doppler Interferometer (MDI) on the Solar \& Heliospheric Observatory (SoHO) have demonstrated that continuous, distortion free, observations of the longitudinal magnetic field from space are very important in our understanding of the Sun's magnetic field. The Solar Optical Telescope (SOT) on the Hinode and the Helioseismic and Magnetic Imager (HMI) on the Solar Dynamics Observatory (SDO) spacecraft will extend our space based observations to include photospheric vector magnetic fields. Although these high resolution observations will greatly advance our knowledge of
Table 1: Comparison of candidate lines that are being developed for current and future space-based photospheric, chromospheric and transition region vector magnetic field measurements.

\begin{tabular}{|c|c|c|c|c|c|}
\hline $\begin{array}{c}\text { Spectral } \\
\text { lines }\end{array}$ & $\begin{array}{c}\lambda \\
(\mathrm{nm})\end{array}$ & $\mathrm{g}$ & $\begin{array}{c}\lambda^{2} \times \mathrm{g} \\
\times 10^{4}\end{array}$ & $\begin{array}{c}\text { Height } \\
(\mathrm{km})\end{array}$ & Remarks \\
\hline \multicolumn{7}{|c|}{ Transition Region } \\
\hline CIV & $\mathbf{1 5 5 . 0}$ & $\mathbf{1 . 2}$ & $\mathbf{2 . 9}$ & $\mathbf{2 2 0 0}$ & SUMI \\
\hline MgII & $\mathbf{2 8 0 . 0}$ & $\mathbf{1 . 2}$ & $\mathbf{9 . 4}$ & $\mathbf{2 0 0 0}$ & SUMI \\
\hline \multicolumn{7}{|c|}{ Chromosphere } \\
\hline CaII & $\mathbf{8 5 4 . 2}$ & 1.1 & $\mathbf{8 0 . 2}$ & 1319 & \\
\hline NaI & 589.5 & 1.3 & 45.1 & 700 & \\
\hline \multicolumn{7}{|c|}{ Photosphere } \\
\hline FeI & 630.2 & 2.5 & 99.2 & 250 & Hinode \\
\hline
\end{tabular}

MTRAP $^{6}$ : The Magnetic Transition Region Probe has all of these lines in its baseline mission.

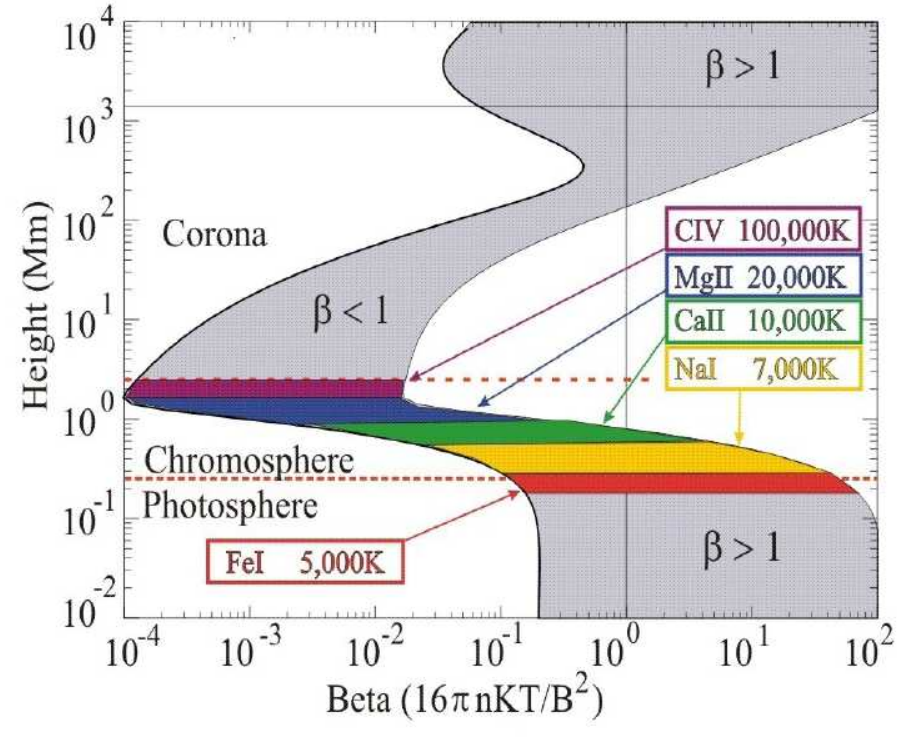

Figure 1. Ratio of gas pressure to magnetic pressure $(\beta)$ as a function of height, for an assumed range of photospheric magnetic field strength of 100 to $2500 \mathrm{G}$ (Gary, 2001) $)^{3}$. 
the behavior of the magnetic field in the photosphere, they will shed little light on how the magnetic field higher in the solar atmosphere transitions to a nearly force-free state. This requires measurement of the field using spectral lines that are formed at temperatures above $20,000{ }^{\circ} \mathrm{K}$. The two spectral lines we have chosen for SUMI are $280 \mathrm{~nm}$ in $\mathrm{MgII}$ and $155 \mathrm{~nm}$ in CIV. These lines occur in the far ultraviolet and are inaccessible from the ground. Together with the lines FeI, $\mathrm{NaI}$ and CaII, they form a quintet of magnetically sensitive lines chosen for a future mission called the Magnetic Transition Region Probe (MTRAP). The demonstration that the MgII and CIV lines can meet the requirements for the force-free region is a prerequisite for this mission and is one of the objectives of the Solar Ultraviolet Magnetograph Investigation (SUMI).

\subsection{Goals for SUMI sounding rocket program}

By the early nineties it was apparent that vector field measurements in the upper chromosphere and transition region were needed, even though this was viewed as a difficult if not impossible task. The Ultraviolet Spectrometer and Polarimeter (UVSP) instrument on board the Solar Maximum Mission (SMM, 1980) was the first space-based instrument to try to map and understand polarization in the transition region. The UVSP observations of MgII suggested that this line was a potential candidate for the upper chromosphere ${ }^{7}$. Although UVSP attempted measurements of the CIV line, poor photon statistics and the loss of the ability to observe the CIV lines early in the mission resulted in a failure to produce convincing evidence that this measurement was possible. While the scientific value of these observations has been acknowledged ${ }^{1,2}$, the measurements are difficult because they are in the vacuum ultraviolet (VUV) wavelength range where the optical efficiency of standard optics is low. The SUMI sounding rocket program was born from development programs ${ }^{8,9,10}$ to improve the overall wavelength and polarization efficiency for the CIV $(155 \mathrm{~nm})$ measurement while including the MgII measurements in its observing program. While these development programs were successful, accommodating the dual-line instrument in a sounding rocket envelope has been challenging.

CIV has always been the driving force in the development of SUMI. This line is formed in the relatively thin transition region which simplifies its interpretation. However the magnetic sensitivity, $\lambda^{2} g$ where $\lambda$ is the wavelength and $g$ is the Landé $g$ factor. The magnetic sensitivity is low compared to lines in the visible and infrared. Table 1 compares the SUMI lines with lines that are being used in photospheric and low chromospheric magnetographs. Certainly the results from SUMI will impact future missions as scientist try to understand the 3-D structure of the Sun's magnetic field.

\section{DESCRIPTION OF SOUNDING ROCKET PAYLOAD}

The SUMI development began as a set of programs to improve the efficiency of polarization measurements in the ultraviolet. As demonstrated by Table 1, polarization measurements in the ultraviolet require high spectral resolution due to the small magnetic sensitivity. Higher spectral resolution is required to resolve the Zeeman splitting. Therefore to reduce the length of the telescope allowing a longer spectrograph (with improved resolution), a cold mirror RitcheyChretien telescope was chosen over a traditional Gregorian telescope (\$3.1). To improve the photon and polarization efficiency, a $\mathrm{MgF}_{2}$ double Wollaston polarizer was selected as the analyzer so that simultaneous measurements of orthogonal polarizations could be made. Ideally, full Stokes polarization measurements, [I, Q, U, V], should be made but photon statistics make CIV linear polarization measurements ( $Q$ and $U$ ) difficult with the limited observing time of a sounding rocket. Therefore the waveplate is optimized for [I, Q, V] measurements in CIV and the full Stokes in MgII (§3.2). Section 3.3 will describe the Toroidal Variable-Line-Space (TVLS) grating technology that is used to achieve the high spectral resolution while reducing the number of optical elements. The last section (§3.4) describes SUMI's cameras. 


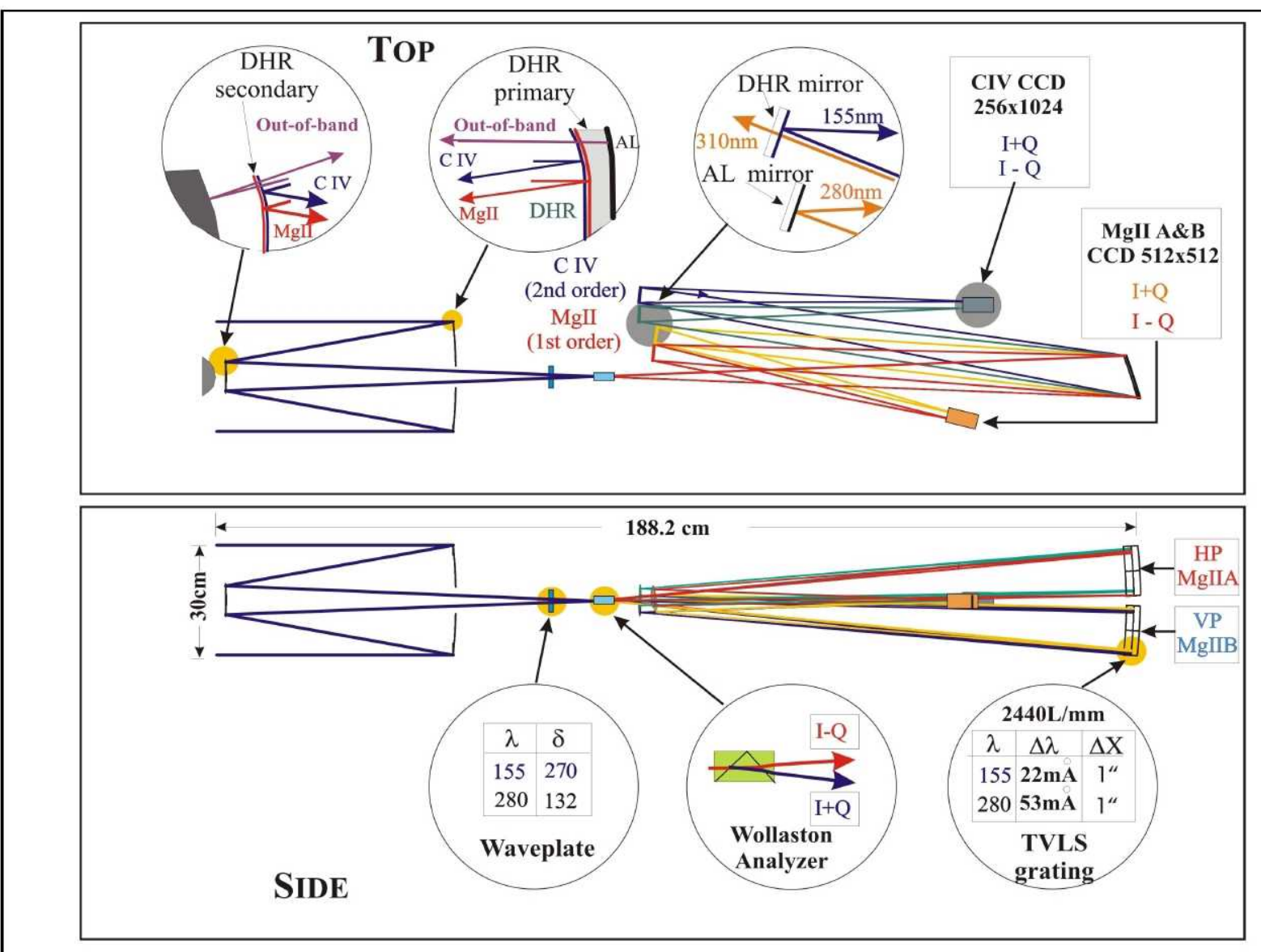

Figure 2. Optical schematic (top view) showing the technologies developed in the SUMI sounding rocket program. In this diagram the CIV wavelength is $155 \mathrm{~nm}(1550 \AA)$ and the MgII is $280 \mathrm{~nm}(2800 \AA)$. The side view of the double Wollaston analyzer is shown and requires two TLVS gratings (designated by the I+Q and I-Q beam paths).

\subsection{Telescope optics}

A solar telescope design must solve the thermal problems associated with direct solar viewing. The simplest solution is a Gregorian telescope with a field stop between the primary and secondary mirrors. While this reduces the thermal load on the secondary by rejecting the unwanted light, the disadvantages are its limited field of view, a longer optical path, and a larger secondary for on-axis designs, which decreases the effective collecting area of the telescope. SUMI's approach for decreasing the thermal load on the secondary mirror is to use a Ritchey-Chretien telescope with special dielectric coatings applied to the front surfaces of both the primary and secondary mirrors (Figure 2). These coatings reflect only the narrow wavelength ranges around the CIV and MgII emission lines which results in a "cold mirror", i.e., a "self-filtering" telescope. The rear surface of the primary mirror is figured and has an aluminum coating that reflects the unwanted radiation back through the telescope and into space. The advantages of this design are that the field of view is not restricted (whole Sun could be imaged by a slitjaw camera) and, for a given instrument length, the smaller telescope size allows a larger spectrograph improving the wavelength resolution. The narrowband UV reflection coatings simplify the thermal environment, minimize infrared and visible light contamination of the spectral data, and act as a blocking filter for the spectrograph. The measured reflectance of these coatings is shown in Figure 3. Although the MgII bandpass may appear off-center, its reflectivity was tuned to minimize the $1^{\text {st }}$ order contamination of $3100 \AA$ light into the CIV measurement, which is made in $2^{\text {nd }}$ order. 


\subsection{Polarimeter and slit jaw camera}

The focal plane optics following the telescope includes the polarimeter, spectrograph slit and slit jaw camera. The polarimeter consists of a $\mathrm{MgF}_{2}$ waveplate and a $\mathrm{MgF}_{2}$ double Wollaston analyzer. Due to the low photon flux at CIV and the weak linear polarization levels, the waveplate was designed to measure circular polarization at $\mathrm{CIV}$ $\left(270^{\circ}\right.$ retardance at $\left.1550 \AA\right)$ and the full Stokes vector at $\mathrm{MgII}\left(132^{\circ} \text { retardance at } 2800 \AA\right)^{9,11}$. During the flight SUMI will concentrate on circular polarization measurements when the payload is above $200 \mathrm{~km}$ (required height for CIV measurements) and will do the linear polarization measurements when the sounding rocket is below $200 \mathrm{~km}$.

The double Wollaston analyzer, which is a polarizing beamsplitter, is the most efficient UV polarizer in this wavelength band ${ }^{9}$. Figure 4 shows the orientation of the double Wollaston and the exiting linearly polarized beams. The orientation of the linear polarization and the polarization

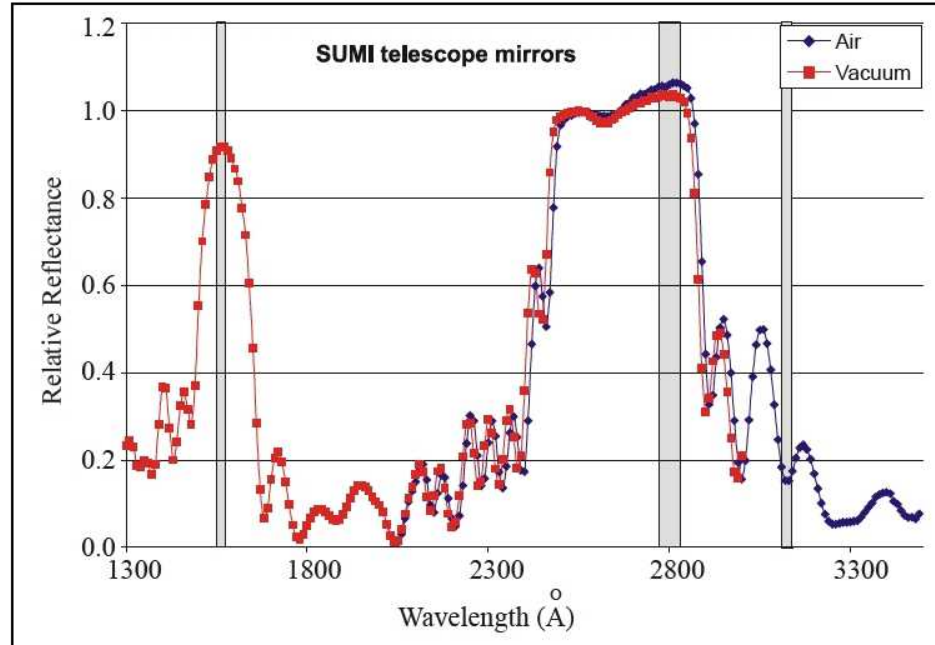

Figure 3. Dielectric High Reflectance (DHR) coating on SUMI primary and secondary telescope mirrors. These coatings act as a prefilters to isolate the CIV $(1550 \AA)$ and $\mathrm{MgII}(2800 \AA)$ lines. The $\mathrm{MgII}$ reflectivity was tuned to minimize the $1^{\text {st }}$ order reflectance of $3100 \AA$ into the $2^{\text {nd }}$ order CIV measurement.

reflectivity of the TVLS gratings were discussed in a previous paper ${ }^{12}$. The selected gratings maximize the reflectance of the vertical linear polarization ( $\mathrm{VP}=+\mathrm{Q}$ defined to be the $\mathrm{E}$ field aligned to the slit, spatial axis), and horizontal linear polarization $(\mathrm{HP}=-\mathrm{Q}$ is perpendicular to slit, dispersion axis).

A dual-beam analyzer is very important in transition region magnetic field measurements since the CIV emission can have rapid changes in intensity ${ }^{18}$. Without simultaneous measurements of orthogonal polarizations, intensity crosstalk can create false signals in SUMI's magnetic field measurements. Since both polarizations are observed, the total transmission is much higher than traditional reflective polarizers even at CIV (the cutoff wavelength of $\mathrm{MgF}_{2}$ is $1150 \AA$ ). Finally, the polarization resolution $\left(<10^{-3}\right)$ is higher and covers a larger wavelength range than traditional reflective analyzers which is important for SUMI's CIV $(1550 \AA)$ and MgII $(2800 \AA)$ measurements. Furthermore, the rotating waveplate allows cross-checks of the image subtraction scheme by flipping the polarization signals from the Sun between the HP3/VP5 detectors. For example, with the waveplate fast axis (FA) at $45^{\circ}$ the VP5 detector would measure $\mathrm{I}+\mathrm{V}$ for $\mathrm{CIV}$ and HP3 I-V; with the FA at $135^{\circ} \mathrm{VP} 5$ would measure I-V and HP3 I+V (see Figure 7 for VP5/HP3 CIV detector definition).

For SUMI's first flight, the slit has a unique structure. The slit is cut into a $6 \mathrm{~mm}$ thick diamond turned aluminum substrate and is $3.7 \mathrm{~mm}$ long ( 3.5 arc minutes) with $0.5 \times 0.5 \mathrm{~mm}$ $(30$ arcsec) boxes on each end. The width of the slit has been split into two sections, a $30 \mu \mathrm{m}(\sim 1.6$ arc seconds) and a $60 \mu \mathrm{m}(\sim 3.2$ arc second $)$. Due to the exploratory nature of the SUMI measurements, the uncertainty in the target at launch and the limited observing time of the flight ( $\sim 6$ minutes), this structure was adopted to give SUMI a larger dynamic range and to capture enough photons to make meaningful polarization measurements at CIV.

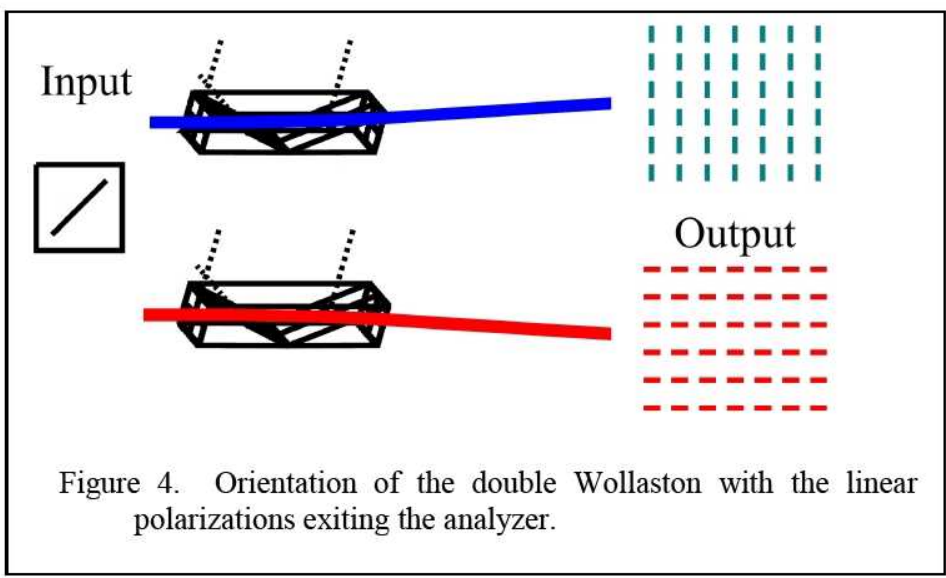


Table 2. Polarization reflectivity measurements for the six gratings fabricated by Jobin Yvon for SUMI: 2007 measurements. The gratings selected for the first flight are \#3 for the HP (HP3) and \#5 for the VP (VP5).

\begin{tabular}{|c|c|c|c|c|c|}
\hline \multirow{2}{*}{ Grating number } & \multirow{2}{*}{ Coating } & \multicolumn{2}{|c|}{ Horizontal Polarization (HP) } & \multicolumn{2}{|c|}{ Vertical Polarization (VP) } \\
\cline { 3 - 6 } & & $\mathrm{CIV}\left(2^{\text {nd }}\right.$ order $)$ & $\mathrm{MgII}\left(1^{\text {st }}\right.$ order $)$ & $\mathrm{CIV}\left(2^{\text {nd }}\right.$ order $)$ & $\mathrm{MgII}\left(1^{\text {st }}\right.$ order $)$ \\
\hline $1 . \mathrm{CY}_{11 \mathrm{~S}}^{*}$ & $\mathrm{AL} / \mathrm{MgF}_{2}$ & $\mathbf{1 2} \%$ & $\mathbf{3 9} \%$ & $29 \%$ & $52 \%$ \\
\hline $2 . \mathrm{CY} 11 \mathrm{O}$ & $\mathrm{AL} / \mathrm{MgF}_{2}$ & $11 \%$ & $33 \%$ & $15 \%$ & $38 \%$ \\
\hline $3 . \mathrm{CY} 11 \mathrm{~N}^{*}$ & $\mathrm{AL} / \mathrm{MgF}_{2}(A L)$ & $\mathbf{1 2} \%(16 \%)$ & $\mathbf{4 1} \%(9 \%)$ & $\mathbf{3 0 \%}(17 \%)$ & $\mathbf{5 4 \%}(49 \%)$ \\
\hline $4 . \mathrm{CY} 11 \mathrm{P}$ & $\mathrm{AL}$ & $16 \%$ & $11 \%$ & $18 \%$ & $52 \%$ \\
\hline $5 . \mathrm{CY} 11 \mathrm{R}$ & $\mathrm{AL}$ & $12 \%$ & $15 \%$ & $\mathbf{2 0 \%}$ & $\mathbf{6 2} \%$ \\
\hline 6. CY11M & $\mathrm{AL}$ & $14 \%$ & $16 \%$ & $17 \%$ & $61 \%$ \\
\hline
\end{tabular}

* Acton $\mathrm{AL} / \mathrm{MgF}_{2}$ coatings. Grating 3 was recoated by Acton after the aluminum measurements showed a low HP reflectance at $\mathrm{MgII}(9 \%)$

The slit-jaw camera is the same E2V CCD used in the MgII measurements (§3.4). This CCD is a frame transfer, backilluminated, uncoated silicon detector array with $512 \times 512$ pixels. The spatial resolution of each pixel has been set to $0.65 "$ which limits the FOV of the slit-jaw camera to 5.6' x 5.6'.

\subsection{Spectrograph}

Since the double Wollaston analyzer produces two polarized images of the slit, two toroidal variable-line-space (TVLS) gratings are required ${ }^{13,14,15}$. The sensitivity of the spectrograph gratings to the polarization exiting the double Wollaston was taken into consideration ${ }^{8}$. In our initial concept a quarterwave plate was placed between the double Wollaston prism and gratings to convert the exiting linear polarization to circular if the gratings were found to be very sensitive to linear polarization. Fortunately, a bare aluminum TVLS grating can be used for the vertical polarization (VP) and a $\mathrm{MgF}_{2}$ coated aluminum grating for the horizontal polarization (HP, see Table 2). Section 4 discusses both the optical modeling and measurements made on these devices.

\subsection{UV cameras}

The SUMI cameras are based on the E2V back illuminated, bare silicon technology. This technology enhances the UV quantum efficiency but at the time SUMI was developed their design was limited to certain CCD structures since they were not part of E2V's standard product line in 2005. For the slit-jaw camera, and the MgII cameras where high speed electronic shuttering was required, a frame transfer $512 \times 512 \mathrm{CCD}$ array with $13 \mu \mathrm{m}$ pixels was selected. The CIV camera uses a full frame $1024 \times 256 \mathrm{CCD}$ array with $26 \mu \mathrm{m}$ pixels. To simplify the electrical wiring between the cameras and the data system, a USB 2.0 interface is used. For ground-based testing and software development, a $\mathrm{MgF}_{2}$ window is mounted in front of all of the cameras. The measured quantum efficiency $(\mathrm{QE})$ for these cameras is $>50 \%{ }^{17}$.

The SUMI sounding rocket has two data systems to control the four USB cameras, the rotating waveplate and to monitor the health and status of the SUMI payload. Each data system has two USB and two Ethernet ports on the processor board. Since the CIV measurements have the highest priority, the CIV data system is the master and drives the timing and telemetry for the SUMI instrument and controls the MgII data system. All of the data from SUMI's cameras is stored on the sounding rocket in a 4GB solid state disk drive attached to each data system. The slit jaw camera is connected to the second USB port on the CIV data system. 


\section{Platinum/Neon Hollow Cathode source CIV: 1548.2 and 1550.7}

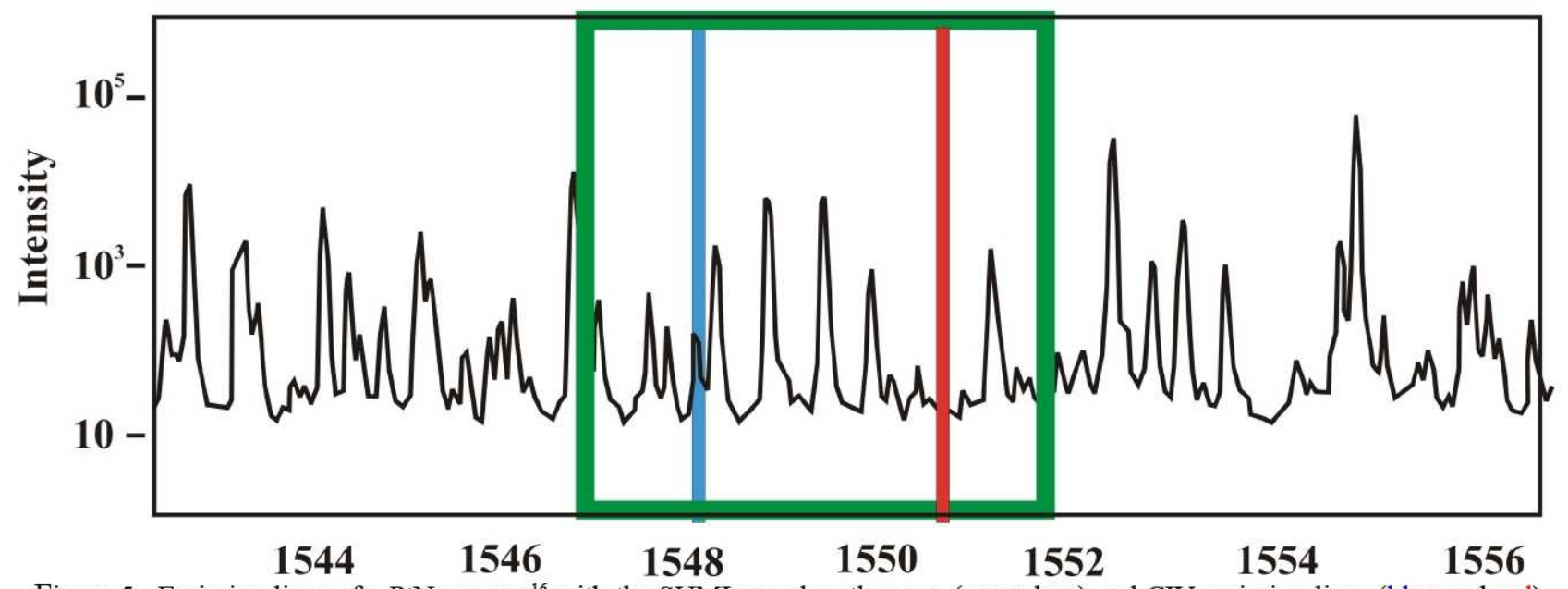

Figure 5. Emission lines of a PtNe source ${ }^{16}$ with the SUMI wavelength range (green box) and CIV emission lines (blue and red) superimposed. The green box is set by the size of the E2V CCD. The $256 \times 26 \mu \mathrm{m}$ pixels represents the wavelength axis of the camera and the $1024 \times 26 \mu \mathrm{m}$ pixels are the spatial/slit axis which captures both polarizations from the double Wollaston (see Figure 7) .

\section{OPTICAL TESTING SUMI}

This section describes the spectral calibration and alignment of SUMI using a Platinum-Neon (PtNe) lamp for the MgII cameras and a carbon spark lamp for the CIV camera. In order to verify the optical alignment, a Zemax model (www.zemax.com) of SUMI was developed that included the wavelengths of the two lamps, the optical efficiency at each wavelength and a model of the spectrograph slit to help in the alignment of the spectrograph cameras and to evaluate the performance of the spectrograph. Section 4.1 provides a short description of the VUV collimator used to test SUMI. Section 4.2 describes the CIV tests, the VUV source and control electronics and compare the Zemax optical model with the CIV measurements. Section 4.3 discusses the MgII tests and modeling.

\subsection{VUV collimator}

Since each optical element represents a loss in photons, especially at CIV, the concept for SUMI was to isolate the CIV and MgII lines using as few optics as possible. This approach led to the development of the dielectric high-reflectivity (DHR) coatings on the telescope mirrors (\$3.1, Figure 3) which act as a dual-line prefilter. While SUMI is efficient in isolating our UV lines, traditional methods to align the instrument cannot be used. Therefore a custom vacuum ultraviolet (VUV collimator) facility had to be developed that was within the budget constraints of our sounding rocket program.

Although the VUV collimator cannot achieve SUMI's resolutions, it can be aligned with traditional instruments since it is an all reflecting system with aluminum $/ \mathrm{MgF}_{2}$ coatings optimize to reach $120 \mathrm{~nm}$. After aligning the VUV collimator, it can be used to provide the vacuum UV wavelengths needed to check the alignment of the SUMI telescope and spectrograph.

Originally, an Acton VM504 monochromator was to provide the spectral calibration for SUMI but SUMI has higher wavelength resolution due to its 2440 lines/mm TVLS gratings compared to the 1200 line/mm grating in the VUV monochromator. With a $+/-0.1 \mathrm{~nm}$ uncertainty in the monochromator's wavelength drive and the lack of a good wavelength reference for the deuterium emission lines, the decision was made to use carbon spark lamp for the CIV alignment (Section 4.2) and the PtNe lamp for the MgII (Section 4.3). 


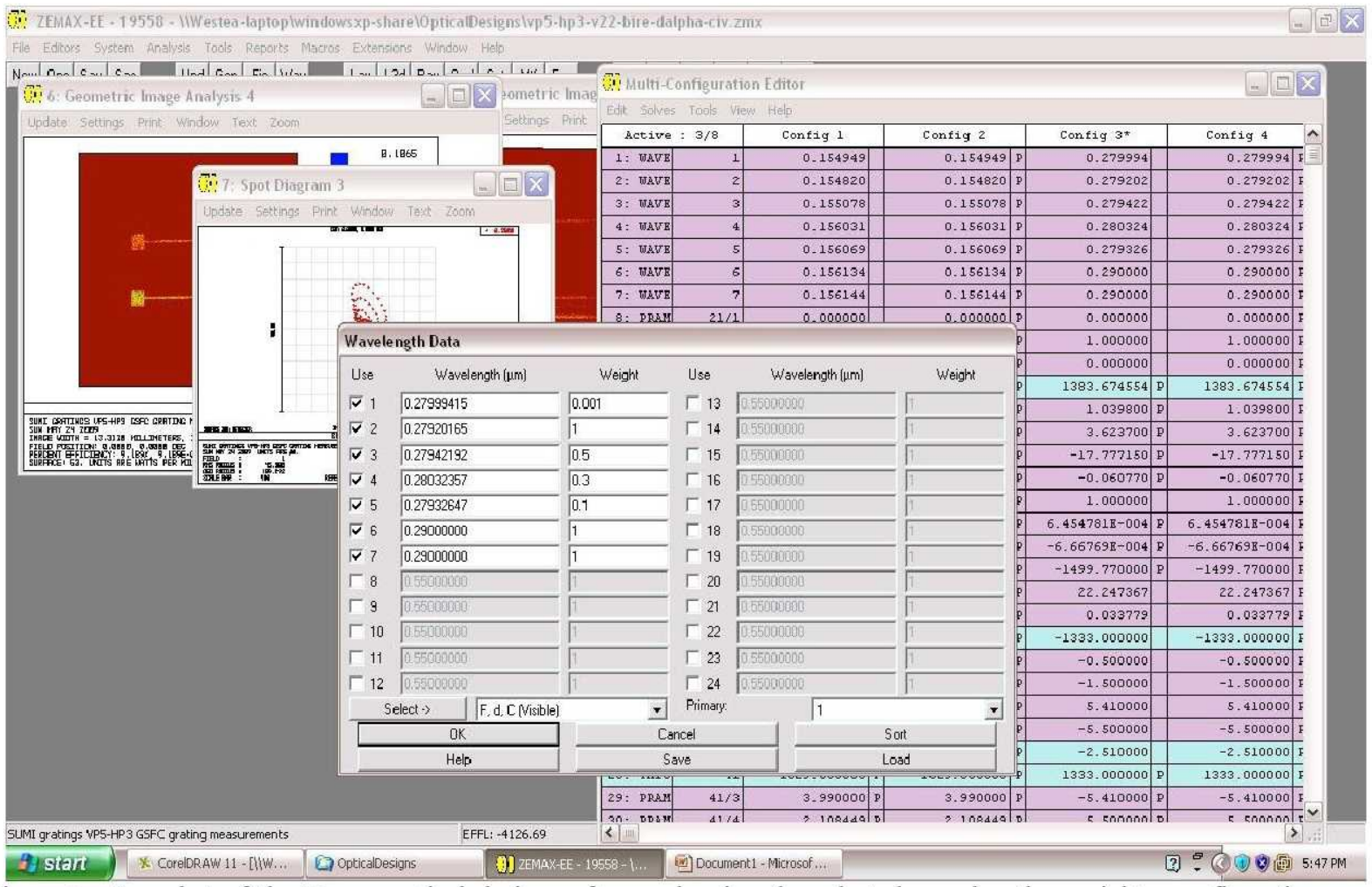

Figure 6. Snapshot of the Zemax optical design software showing the selected wavelengths, weights, configurations and Geometric Image Analysis screens.
VP5 HP3
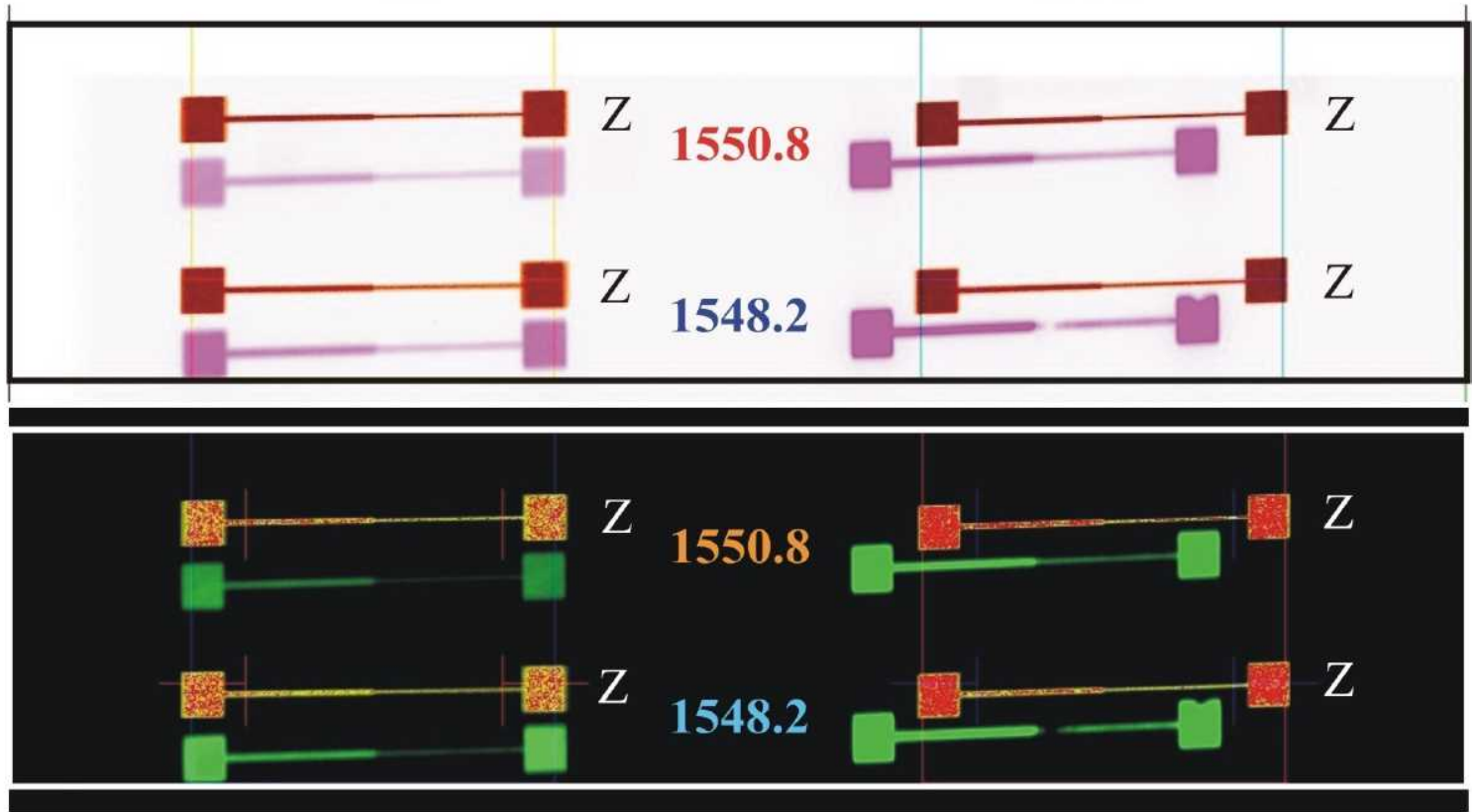

Figure 7. SUMI display screen showing an overlay of the Zemax spectra (Labeled Z) onto an image from the CIV camera. The image on the top is the negative of the lower image. The CIV camera images both the horizontal (HP3) and vertical (VP5) gratings. The light purple in top image (green in lower) are images of the carbon spark lamp. 


\subsection{CIV alignment}

Figure 5 shows the PtNe source ${ }^{16}$ in the CIV wavelength range. Certainly there are several strong emission peaks (intensity $>10^{4}$ ) near the CIV lines (red and blue lines in Figure 5) but scanning across this range while working in a vacuum and keeping the TVLS gratings centered onto the CIV detector to identify these lines would be difficult. Also, PtNe emission lines below the $10^{4}$ level require long exposure times (>10s) and the Zemax CIV model would have to include a large number of these lines for comparisons between the optical model and spectrograph measurements. Fortunately, NIST had a carbon sparks lamp that could provide the two strong CIV emission lines that were needed to align the TVLS gratings. By using this source, the identification and alignment of the PtNe emission lines within the MgII was simplified (Section 4.3).

The basic construction of the NIST carbon sliding spark light source is that of two (graphite) electrodes that are in good mechanical contact with a (quartz) insulator. This provides a surface between the electrodes along which the spark can be initiated. A high voltage power supply controls voltage across the gap and a rotating auxiliary spark gap in air supplies the trigger. Peak current shape is modified by an external inductor (i.e., number of turns) and the voltage to spark lamp. The peak current is then fined-tuned by a contact carbon plate resistor ${ }^{19,20}$.

To help in the alignment process, a Zemax model was developed with the primary wavelengths near the CIV (and MgII) wavelength band and spectrograph custom slit. The weight parameter in the wavelength data is used to simulate the intensity differences of the various lines (see Figure 6 Wavelength Data table). Since the first wavelength is always centered in the Geometric Image routine, a dummy line with a weight of 0.001 is created in that position in the table. That wavelength is average of the two CIV (or MgII) emission lines. With this setup, a Geometric Image Analysis (GIA) file can be created to show all of the emission lines listed in the wavelength table (see images in the background of Figure 6). The field of view of the Zemax model is set to be twice that of the spectrograph cameras so that nearby emission lines can be seen and compared to the images from the cameras. While this larger field of view is not important in the CIV alignment using the carbon lamp, the larger field of view helps in the identification of the PtNe lines seen by the MgII cameras (Section 4.3). Figure 6 shows a snapshot of the Zemax optical model that was used to create the GIA images. To help in the alignment of the TVLS gratings, a special display program was developed to overlay the GIA images and the spectrograph images. This is especially helpful in the MgII alignment where the cameras have a small field of view in both the spectral and spatial axes.

Figure 7 shows an overlay of the Zemax model with the images from the CIV camera. In normal operation the CIV spectra are stored into the green memory plane of the display while the Zemax model is stored in the red and blue

\section{Platinum/Neon Hollow Cathode source Mgll: 2796.35 and 2803.53A}

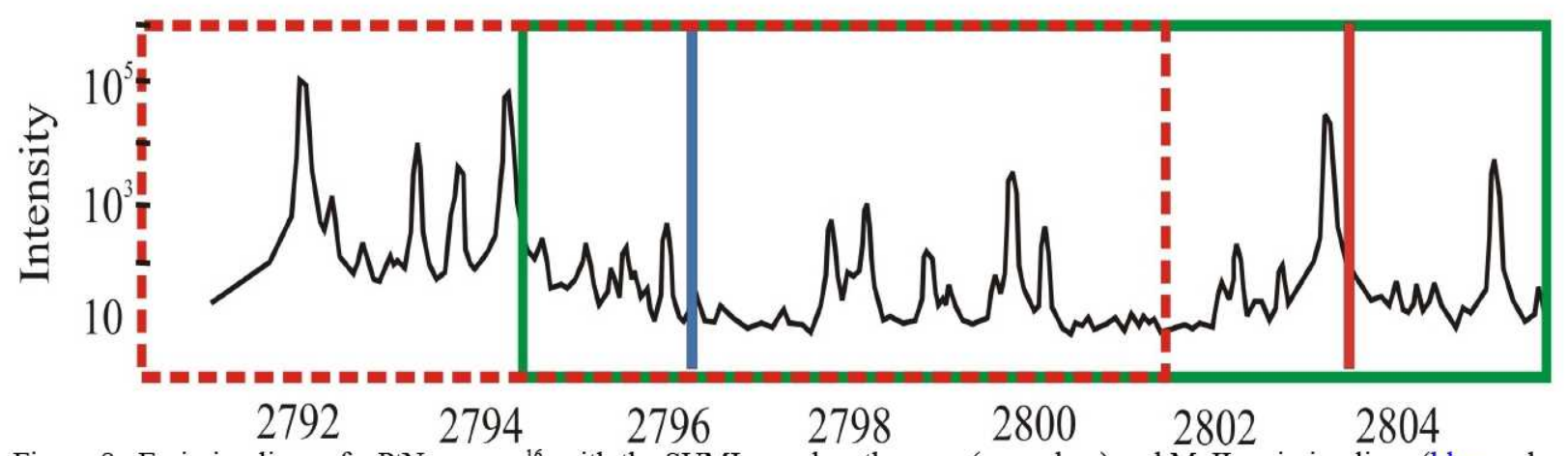

Figure 8. Emission lines of a PtNe source ${ }^{16}$ with the SUMI wavelength range (green box) and MgII emission lines (blue and red) superimposed. The green box represents the correct alignment and the dashed red box the alignment shown in Figure 9. The size of the green box is set by the $512 \times 13 \mu \mathrm{m}$ pixels of the E2V camera. 


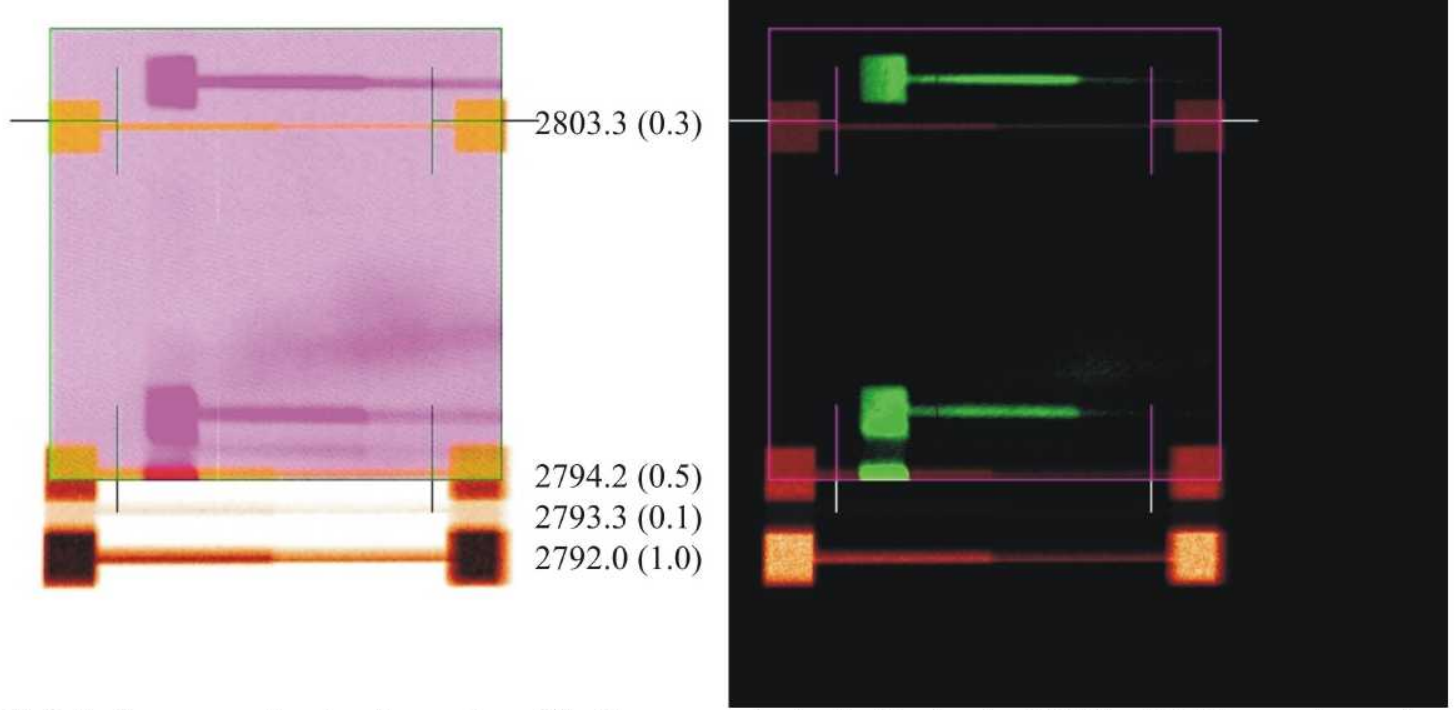

Figure 9. SUMI display screen showing the overlay of the Zemax spectra (red/yellow) onto SUMI's MgIIA spectrograph camera. The FOV of the Zemax model is twice the field of view of the MgIIA camera to help identify the PtNe lines. The image on the left is negative of the right image (normal mode). The lines crossing the Zemax PtNe lines are used in the alignment of the test images with the Zemax model. The emission lines that are listed between the two images are the PtNe lines. The MgII lines are listed in the top left with the midpoint wavelength in parenthesis.

planes (lower image in Figure 7). Additional line plots (not shown) are used to align the CIV spectra onto to Zemax model. These locations are indicated by the lines added to the Zemax model.

\subsection{MgII alignment}

Figure 8 shows the PtNe source ${ }^{16}$ in the MgII wavelength range. The strong lines that can easily be resolved in the MgII cameras have intensities $>10^{4}$. The MgII lines (red and blue lines in Figure 8 ) are overlaid onto the PtNe spectrum with the green solid box indicating the wavelength spectral range of the MgI cameras. The red dashed box was added to show the wavelength seen in the Figure 9 images.

Since SUMI's design was for a sounding rocket flight environment, the mechanical ability to scan through the PtNe spectra to identify the emission lines is very restricted. Therefore, the use of the carbon lamp with its strong CIV emission lines helped by allowing us to quickly identify those lines and adjust the position and tilt of the two TVLS gratings. The final step for the MgII alignment is to confirm the identity of the PtNe emission lines and use the fold flats to position those lines to their correct position with reference to the Zemax modeling.

Figure 9 shows the Zemax model with its alignment grid and the PtNe lines observed by the MgIIA (HP3) camera. When correctly aligned the right overlay plot should show "white" slit images (black on the right image). Since the Zemax model also tries to match the intensity of the PtNe lines (using the Weight parameter), the real-time test images will be a shade of gray. 


\section{SUMMARY}

Since each optical element represents a loss in photons, especially at CIV, the concept for SUMI was to isolate the CIV and MgII lines using as few optics as possible. This approach led to the development of the dielectric high-reflectivity (DHR) coatings on the telescope mirrors ( $\$ 3.1$, Figure 3) which act as a dual-line prefilter for the spectrograph while limiting the thermal load on the secondary mirror. A beamsplitting double Wollaston analyzer (\$3.2) doubles the efficiency by allowing simultaneous measurements of orthogonal polarizations. Finally the TVLS gratings provides both the wavelength resolution and imaging optics for the scientific cameras. While SUMI is efficient in isolating our UV lines, traditional methods to align the instrument can not be used. Therefore a custom vacuum ultraviolet VUV collimator had to be developed that was within the budget constraints of our sounding rocket program while providing the data required to verify that SUMI could achieve the spatial, wavelength and polarization resolution necessary to meet its mission goals. Using this collimator with a carbon sliding spark lamp (§4.2) we were able to align the CIV emission lines onto our CIV spectrograph camera. With the CIV lines in their correct position, the identification and alignment of the PtNe lines that fall within the wavelength band of the MgII cameras was greatly simplified. 


\section{REFERENCES}

[1] H. Uitenbroek, "Multilevel Radiative Transfer with Partial Frequency Redistribution," Astrophysical Journal, 557, 389-398 (2001).

[2] H. Peter, "On the nature of the transition region from the chromosphere to the corona of the Sun," $A \& A, 374$, $1108-1120$ (2001).

[3] G. A. Gary, "Plasma Beta above a Solar Active Region: Rethinking the Paradigm," Solar Phys., 203, 71 (2001).

[4] R. Rosner, B. C. Low, and T. E. Holzer, "Physical processes in the solar corona," Physics of the sun, D. Reidel Publishing Co, 135-180 (1986).

[5] Moore, Davis, and Hathaway, "High-Resolution Solar Magnetography from Space: Beyond Solar-B," http://solarscience.msfc.nasa.gov/Beyond Solar-B.shtml, (2001)

[6] J. Davis, E. West, R. Moore, G. Gary K. Kobayashi, J. Oberright, D. Evans, H. Wood, J. L. R. Saba and D. Alexander, "MTRAP: The Magnetic Transition Region Probe," SPIE: Solar Physics and Space Weather Instrumentation. 5901, 1-12 (2005).

[7] Gurman, J. B., "The MG II H line in sunspot umbrae," Solar Phys., 90, 13-15 (1984).

[8] West, E. A., J. G. Porter, J. M. Davis, G. A. Gary, D. M. Rabin, R. J. Thomas, and J. M. Davila, "Overview of the Solar Ultraviolet Magnetograph Investigation," SPIE: Instrumentation for UV/EUV Astronomy and Solar Missions, 4139, 350-361 (2000).

[9] West, E. A., J. G. Porter, J. M. Davis, G. A. Gary, and M. Adams, "Development of a polarimeter for magnetic field measurements in the ultraviolet," SPIE: Polarization Analysis: Measurement and Remote Sensing IV, 4481, pp. 109-117 (2001).

[10] West, E. A., J. G. Porter, J. M. Davis, G. A. Gary, M. Adams, S. Smith and J. F. Hraba, "Optical characteristics of the Marshall Space Flight Center Solar Ultraviolet Magnetograph," SPIE: UV/EUV and Visible Space Instrumentation for Astronmy and Solar Physics, 4498, 101-110 (2001).

[11] K. Kobayashi, E. A. West and M. Noble, "Polarization measurements in the Vacuum Ultraviolet," SPIE: Polarization Science and Remote Sensing II. 5888, 1-12 (2005).

[12] K. Kobayashi, E. A. West, J. M. Davis and G. A. Gary, "Polarization measurements on SUMI's TVLS gratings", SPIE: Polarization Science and Remote Sensing III. 6682, 1-12 (2007).

[13] T. Kita and T. Harada, "Use of Aberration-Corrected Concave Gratings in Optical Demultiplexers," Applied Optics, 22, 819-825 (1983).

[14] R. J. Thomas, "Toroidal varied line-space (TVLS) gratings," SPIE: Innovative Telescopes and Instrumentation for Solar Astorphysics, 4853, 411-418 (2003).

[15] L. Poletto and R. J. Thomas, "Stigmatic Spectrometers for Extended Sources: Design with Toroidal Varied Line-Space (TVLS) Gratings," Applied Optics, 43, 2029-2038 (2004).

[16] NIST, "Platinum Neon Data," http://physics.nist.gov/PhysRefData/platinum/contents.html , 1-2 (2008)

[17] E. A. West, J. G. Porter, J. M. Davis, G. A. Gary, M. W. Noble, M. Lewis and Roger J. Thomas, "The Marshall Space Flight Center Solar Ultraviolet Magnetograph," SPIE: UV and Gamma-Ray Space Telescope Systems, 5488, 801-812 (2004).

[18] J. G. Porter, J. M. Fontenla and G. M. Simnett, "Simultaneous ultraviolet and X-ray observations of solar microflares," Astrophysical Journal, 438, vol. 1, 472-479 (1995).

[19] Bochkasten, K., "A study of CIII by Means of a Sliding Vacuum Spark," Arriv for Fysik, 9, 457 (1955).

[20] J. Reader, G. L. Epstein, and J. O. Ekberg, "Spectra of Rb II, Sr III, Y IV, Zr V, Nb VI, and Mo VII in the vacuum ultraviolet," J. Opt. Soc. Am. 62, 273-284 (1972). 